\title{
Cavity method for quantum spin glasses on the Bethe lattice
}

\author{
C. Laumann, A. Scardicchio, and S. L. Sondhi \\ Department of Physics, Joseph Henry Laboratories, \\ Princeton University, Princeton NJ 08544 and \\ Princeton Center for Theoretical Physics, \\ Princeton University, Princeton NJ 08544
}

\begin{abstract}
We propose a generalization of the cavity method to quantum spin glasses on fixed connectivity lattices. Our work is motivated by the recent refinements of the classical technique and its potential application to quantum computational problems. We numerically solve for the phase structure of a connectivity $q=3$ transverse field Ising model on a Bethe lattice with $\pm J$ couplings, and investigate the distribution of various classical and quantum observables.
\end{abstract}

PACS numbers: 75.50.Lk, 05.30.-d 


\section{INTRODUCTION}

The appearance of finite connectivity trees (eg Cayley trees, Bethe lattices) in the study of spin glasses has a long history that goes back to the first papers on the SherringtonKirkpatrick model $\underline{1}^{1} 2$. Thouless, Anderson and Palmer ${ }^{2}$ showed by means of a diagrammatic expansion of the partition function that in the spin-glass phase the mean-field theory is defined by the diagrams which describe an infinite tree with connectivity $q \gg 1$. They then wrote the mean field equations ${ }^{33}$ for such a tree and simplified the results in the small coupling, large connectivity regime (since in the SK model $q=N$ and the couplings $\left.J_{i j} \propto 1 / \sqrt{N}\right)$. Those mean field equations are known as the TAP equations and the peculiar characteristics of their solutions, in particular their large number $\underline{3}$, were an important indicator of the complexity of the spin glass phase that arose in parallel with the remarkable developments in the study of the replicated free energy $\underline{4}, \underline{5}$.

Many authors have since studied the problem of a spin glass on the Bethe lattice, $7,8,9,10,11$ with two distinct motivations. The first of these has been to attempt to find a model of a short ranged spin glass where one can rigorously assess Parisi's picture for the organization of the Gibbs states in short-range systems $\frac{12}{}$. The second has come from computer science whereby a set of optimization problems can be recast as frustrated problems on random graphs with the local connectivity of a tree $\underline{13}$.

Despite much work, the Bethe lattice has not yielded a decisive verdict for or against the Parisi picture of a multitude of Gibbs states in the ordered phase of a spin glass although the case for it is perhaps stronger here than on regular lattices with short ranged interactions. This is tied up with the question of defining the infinite Bethe lattice limit starting from finite graphs. It is possible to do so either via a sequence of Cayley trees with random, frustrating, boundary conditions or via a sequence of random graphs with fixed connectivity. The latter sequence has frustrating loops of typical size diverging $O(\log (N) / \log (q-1))$ for a graph with $N$ points and connectivity $q$ and thus locally looks like a tree $\stackrel{14}{~}$. The Cayley tree sequence can always be analyzed in terms of a recursion relation that we review below and does not appear to lead to the Parisi structure ${ }^{9}$. However, it has been argued that the random graph problem is fundamentally different and does lead to replica symmetry breaking 15 .

Happily, the perspective provided by spin glass theory on optimization problems has been quite fruitful regardless. Starting in the early 80 's $\frac{16,17}{17}$, it became clear that much was gained 
by the recognition that various optimization problems in computer science were equivalent to finding ground states of certain statistical mechanics problems. A typical example of this connection is given by the k-SAT problem, which asks "Given a boolean expression $J$ on $N$ bits $\sigma_{i}$ composed of the conjunction of $M$ clauses, each of which involves exactly $k$ of the bits, is it satisfiable by some bit assignment?" This can be recast in Hamiltonian form by writing a cost function $H_{J}\left[\left\{\sigma_{i}\right\}\right]$ that evaluates the number of violated clauses. In this language, the bits naturally become Ising spins, the expression $J$ becomes a particular instance of some spin glass and the original question requires determining the ground state energy. The large $N$ limit is a problem in statistical mechanics and typical-case analysis for k-SAT becomes the disorder averaged analysis of spin glass theory. In this limit, $k$-SAT develops several phase transitions as a function of $\alpha=M / N$, the number of clauses per bit $\underline{18}$. With increasing $\alpha$, the most salient features are that the problem goes from being easily solved and satisfiable, to an intermediate glassy phase with many local ground states, to a typically unsatisfiable phase where the ground state energy density is positive.

A key role in these developments has been played by the so called cavity method - a complex of analytical and numerical techniques refined recently $\underline{\underline{13}}, \underline{15}$ for studying classical spin glasses on tree-like graphs. Applied to the k-SAT problem, the cavity method suggests the above phase diagram and provides numerical values for its critical points. Moreover, the technique can be applied to a particular instance of k-SAT and the information so obtained about the free energy landscape now guides the search procedure in state-of-the-art k-SAT algorithms 13 .

In this paper we turn to quantum spin glasses on Bethe lattices, specifically to the problem of extending the cavity method to their analysis. As in the classical case, there are two distinct reasons to be interested in these systems. There is the intrinsic interest of the interplay between quantum mechanics and spin glass behavior, about which the difficulties of the classical case serve as both caution and enticement. A second motivation now arises from the rapid recent developments in quantum computing. In particular, the discovery of the ground state of a classical spin system $H_{J}$ derived from a computational problem is ideally suited to solution by the adiabatic algorithm $\underline{19}^{\underline{1}}$, which allows a quantum computer to solve such a problem by means of an adiabatic change of the parameters in the Hamiltonian. The adiabatic algorithm would trace a path in operator space starting from a simpler Hamiltonian $H_{0}$ and ending at $H_{J}$. For instance, $H_{0}$ could be the Hamiltonian for $N$ independent spins 
in a large transverse magnetic field $B_{t}$ and the path could be to slowly lower the field and raise the Ising couplings $J$. Starting from the easily found ground state of $H_{0}$ a sufficiently slow evolution will carry the system to the ground state of $H_{J}$. The power of this algorithm is therefore measured in the scaling of the evolution time with the number of variables $N$.

This discussion certainly suggests that a careful study of the phase transitions encountered on the path between $H_{0}$ and $H_{J}$ is in order. However, the necessity of understanding the "deep" quantum spin glass phase far from the phase transition is also clear. The structure of this phase, especially its nontrivial energy landscape, is probably as important as the nature of the phase transition. In this paper we take a first step toward understanding the quantum spin glass phase by generalizing the cavity method used to study the classical problem. Compared to the replica method or direct study of the quantum TAP equations, we believe the cavity method outlined in this paper provides much more physically transparent information about the SG phase. It also may be applied to many other quantum phase transitions on the Bethe lattice, whether induced by disorder or more typical symmetry breaking.

We note that the topic of quantum spin glasses on trees has been tackled before in the literature from the point of view of statistical mechanics ${ }^{20}$ and computation theory ${ }^{21}$. However, in order to proceed analytically and due to the complexity of the problem, these works have employed several uncontrolled approximations. We will compare with them briefly in the core of the paper. While we have used approximations in our (numerical) analysis as well, we believe they are better controlled, since we show how to systematically improve them and how the results are robust with respect to the improvements. More evolved computational methods for tackling very similar problems have appeared in the literature on dynamical mean field theory ${ }^{22}$ and could possibly be applied to the quantum cavity method on glasses. These methods may allow a study of the zero temperature case, which our approximations could not capture.

Let us turn now to a very brief overview of the results in this paper. We first formulate the cavity method for the transverse Ising spin glass on a Bethe lattice. This is a Markov process for the on-site effective action whose stationary probability distribution can be found numerically using a population dynamics algorithm. This will be the main result of the paper. In this way we obtain the phase boundary in the $\left(B_{t}, T\right)$ plane, the values of the usual thermodynamic quantities (free energy, energy, entropy and $q_{E A}$, the Edward-Anderson 
order parameter ${ }^{23}$ ) and more typical 'quantum' quantities like the single spin von Neumann entropy. Indeed, from the fixed point probability distributions for the effective actions we can calculate distributions for all of the statistical properties of the system. This leaves much room for further work.

The plan of the paper is the following: we introduce the classical cavity method for uniform ferromagnetic systems and spin glasses in Section III we propose its generalization to the quantum spin glass problem in III, we apply it to the numerical study of a Bethe lattice with connectivity three in IV. Discussion and directions for further work will be presented in the last sections.

\section{CLASSICAL CAVITY METHOD}

The cavity method is a way of finding the spin glass free energy by means of selfconsistency equations for the probability distributions of quantities characterizing the statistics of the spins (the cavity fields). It has several virtues compared with the replica method, in particular if applied to spin glasses with finite connectivity. We will return to them after we have explained how the cavity method works.

\section{A. The Bethe-Peierls method for the ferromagnet}

For conceptual clarity we will first recall the "cavity method" in its simplest form as applied to a ferromagnetic Ising model living on a Bethe lattice with connectivity $q$. As mentioned above, there is considerable subtlety in defining a sensible infinite tree model, but for this simple case, a sequence of Cayley trees with uniform small boundary fields to break the Ising symmetry will suffice. In any event, we shall focus here on the local structure of the model.

The classical Hamiltonian is

$$
H=-\sum_{(i j)} J_{i j} \sigma_{i} \sigma_{j}
$$

where $\sigma_{i} \in\{ \pm 1\}$ are Ising spins, $J_{i j}=J>0$ is a uniform ferromagnetic coupling and the sum is over bonds in the Bethe lattice. To create a cavity, we pick a spin $\sigma_{0}$ in the Bethe 
lattice and imagine removing it:

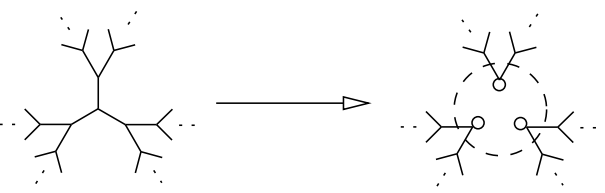

Each of $\sigma_{0}$ 's neighbors is a cavity spin, connected to $q-1$ spins and sitting at the root of a branch of the original tree. Notice that in the absence of $\sigma_{0}$ these $q$ branches are entirely independent. Similarly, a cavity spin $\sigma_{1}$ mediates the only interaction between the $q-1$ branches sitting above it.

We can define three important operations we can perform on graphs with cavity spins: iteration, merging, and link addition (Fig. 1.) For our immediate purposes, the most important of these is iteration, which takes $q-1$ rooted branches of depth $l$ and links them into a single new branch of depth $l+1$ with a new cavity spin at the root. Thus we can construct an arbitrarily large Cayley tree by iterating inward from its boundary spins many times followed by a final merge operation to form the center.

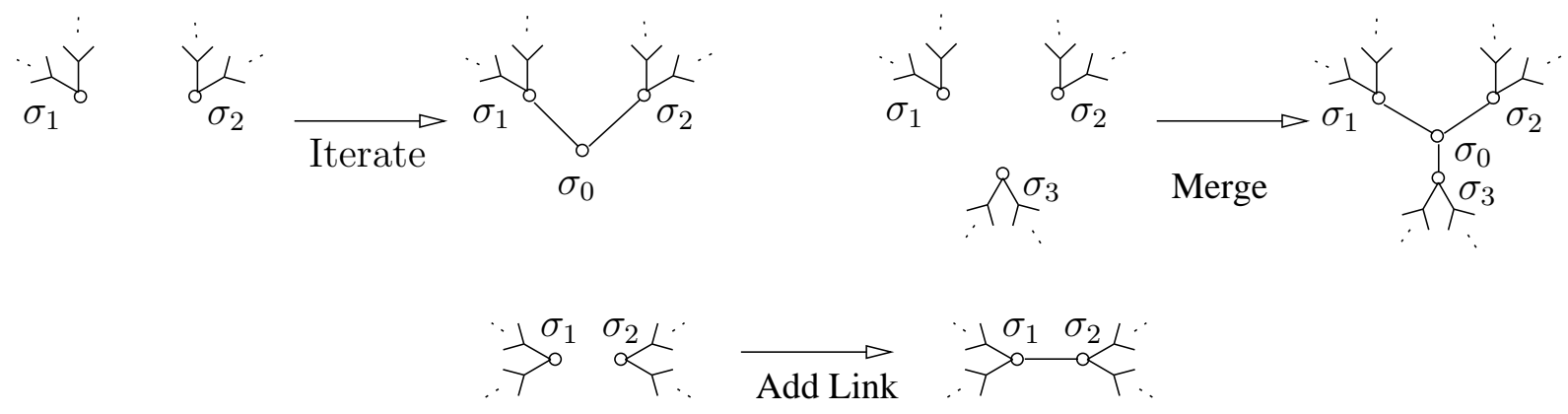

FIG. 1: The three cavity operations. Although we have not labeled them in the Figure, the new links are $J_{01}, J_{02}$, etc $\ldots$

Consider the iteration operation: the added spin $\sigma_{0}$ receives thermodynamic information regarding each of the $q-1$ branches only through the thermal distribution of the cavity spins $\sigma_{1}, \ldots, \sigma_{q-1}$. In the absence of $\sigma_{0}$, each of these Ising variables has independent statistics characterized fully by its thermal probability distribution:

$$
\psi_{i}\left(\sigma_{i}\right)=\frac{e^{\beta h_{i} \sigma_{i}}}{2 \cosh \left(\beta h_{i}\right)} .
$$

which defines the cavity field $h_{i}$. Since $\sigma_{1} \in\{ \pm 1\}$, there are only two possible configurations of the spin and only $2-1=1$ real numbers are needed to characterize the probability 
distribution. In this sense, the cavity field $h_{i}$ is merely a good parameterization of the distribution $\psi_{i}\left(\sigma_{i}\right)$. We emphasize this viewpoint because it will naturally generalize to the quantum case.

We introduce a simple graphical convention for cavity spins:

$$
\forall \dot{\gamma}=\xi
$$

The open circle indicates a spin variable and the wiggly line indicates the effective field attached to it. With this notation, an iteration operation can be represented:

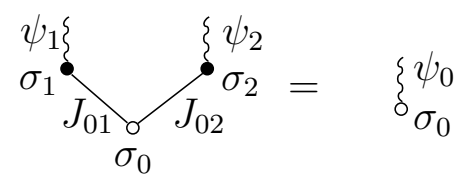

where the filled circle indicates summing out a spin variable. More formally, the state of the spin $\sigma_{0}$ depends on the state of the $q-1$ spins as

$$
\psi_{0}\left(\sigma_{0}\right)=\frac{1}{Z} \sum_{\sigma_{1}, \ldots, \sigma_{q-1}= \pm 1} \exp \left(\beta \sum_{i} J_{0 i} \sigma_{0} \sigma_{i}\right) \psi_{1}\left(\sigma_{1}\right) \ldots \psi_{q-1}\left(\sigma_{q-1}\right)
$$

where $Z$ is a normalization factor so $\sum_{\sigma_{0}} \psi\left(\sigma_{0}\right)=1$. In terms of cavity fields this equation implies

$$
h_{0}=\frac{1}{\beta} \sum_{i=1}^{q-1} \tanh ^{-1}\left(\tanh \left(\beta J_{0 i}\right) \tanh \left(\beta h_{i}\right)\right) \equiv U\left(\left\{h_{i}\right\},\left\{J_{0 i}\right\}\right) .
$$

Finally, to solve the ferromagnet, we note that all the $J_{i j}=J$ and apply uniform boundary fields (say slightly positive) to the Cayley tree "leaves". From this uniform starting point, we expect to find fixed points for the cavity fields under iteration given by

$$
h=\frac{q-1}{\beta} \tanh ^{-1}(\tanh (\beta J) \tanh (\beta h))
$$

This is precisely the Bethe-Peierls self-consistency equation for a mean field ferromagnetic in a lattice of coordination number $q$.

\section{B. Classical Spin Glass on a Cayley Tree}

The careful reader will have noticed that until Eq. (8), we did not anywhere exploit the uniformity of $J_{i j}$ or $h_{i}$ in the foregoing discussion. With this foundation laid, we can make 
short work of the classical Bethe lattice Ising spin glass. Again, we consider a Bethe lattice defined as a limit of Cayley trees with fixed boundary conditions rather than as a limit of random graphs. The Hamiltonian is now

$$
H_{J}=-\sum_{(i j)} J_{i j} \sigma_{i} \sigma_{j}
$$

where the $J_{i j}$ are i.i.d. random variables drawn from some distribution $P(J)$. For simplicity and because of its connection to computational problems, we will restrict our attention to the $\pm J$ model

$$
P\left(J_{i j}\right)=\frac{1}{2} \delta\left(J_{i j}-J\right)+\frac{1}{2} \delta\left(J_{i j}+J\right)
$$

although much of the discussion has broader validity.

The iteration equation (6) is still valid for particular realizations of the $J$, but since these are random variables, it now defines a Markov process for the cavity fields. Throughout the graph, these fields will be site dependent random variables, but deep inside the tree, they ought to be distributed according to a probability distribution $P(h)$ that represents a fixed point of the Markov process $\underline{34}$ This fixed point distribution will satisfy

$$
P(h)=\int \prod_{i=1}^{q-1} d h_{i} P\left(h_{i}\right)\left\langle\delta\left(h-U\left(\left\{h_{i}\right\},\left\{J_{0 i}\right\}\right)\right\rangle_{J} .\right.
$$

In terms of the spin distribution, this becomes the functional equation

$$
P[\psi]=\int\left(\prod_{i=1}^{q-1} D \psi_{i} P\left[\psi_{i}\right]\right)\left\langle\delta\left[\psi(\sigma)-\psi_{0}\left(\sigma ;\left\{\psi_{i}\right\},\left\{J_{0 i}\right\}\right)\right]\right\rangle_{J}
$$

where $\psi_{0}(\sigma)$ is given by Eq. (6) .

This distribution is the order parameter for the spin glass. It is a $\delta$ function at $h=0$ in the high temperature phase. As the temperature is lowered, $P(h)$ broadens to have finite support below some mean field-like phase transition. Defining $\tau=T / J$, this phase transition is located at $\tau_{c}=1 / \tanh ^{-1}\left(\frac{1}{\sqrt{q-1}}\right)$. It is possible to write the free energy per site in terms of $P(h)$ as

$$
\begin{aligned}
F & =\int \prod_{i=1}^{q} d h_{i} P\left(h_{i}\right) F_{q+1}-\frac{q}{2} \int \prod_{i=1}^{2} d h_{i} P\left(h_{i}\right) F_{2} \\
F_{q+1} & =-\frac{1}{\beta}\left\langle\ln \sum_{\sigma_{0}, \sigma_{1}, \ldots, \sigma_{q}} \exp \left(\beta \sum_{i=1}^{q} J_{0 i} \sigma_{0} \sigma_{i}\right) \prod_{i=1}^{q} \psi_{i}\left(\sigma_{i}\right)\right\rangle_{J, \psi} \\
F_{2} & =-\frac{1}{\beta}\left\langle\ln \sum_{\sigma_{1}, \sigma_{2}} \exp \left(\beta J_{12} \sigma_{1} \sigma_{2}\right) \psi_{1}\left(\sigma_{1}\right) \psi_{2}\left(\sigma_{2}\right)\right\rangle_{J, \psi} .
\end{aligned}
$$


This rather complicated looking expression is actually simply the average change in free energy due to a merge operation minus $\frac{q}{2}$ times the average change in free energy due to a link addition. Graphically,

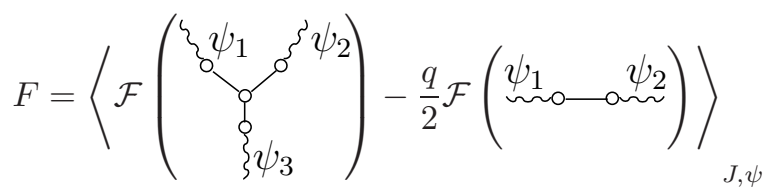

where $\mathcal{F}$ of a diagram is the free energy of a system with spin variables given by the unfilled circles.

It is possible to see that if $P$ satisfies (11) then $\delta F / \delta P(h)=0$. Other expressions for the free energy $F$ have appeared in the literature but it has been shown that they are all equivalent to each other, if the consistency equation (11) is verified. The role of the free energy in the cavity method is secondary as one does not solve the variational problem, as in the replica method, by working on the free energy directly. Rather, one finds the probability distribution $P$ by analytical or numerical methods and then derives all of the statistical observables from $P . \underline{35}$ The equivalence of the two formulations has been put forward in Ref. 11,15 and in many other works.

We have up to this point assumed that the underlying lattice is in fact a tree and that the removal of a spin to create a cavity completely decouples the neighboring branches. On such models, the cavity method we have described is exact. An important generalization of the cavity method arises in its heuristic application to locally tree-like random graphs where the typical loop size diverges logarithmically and any finite neighborhood is a simple tree. In this case however, the decoupling of cavity spins is not exact and it is necessary to introduce the so-called "replica symmetry breaking" ansatz on the structure of thermodynamic states in order to correctly describe the frozen (glassy) phase. Although this is a vital component of the modern understanding of such glasses, we have not included it in our quantum treatment below because we believe their is still much to be understood about the quantum model on the much simpler trees. We refer the interested reader to Ref. 15 for a more detailed description of replica symmetry breaking in the classical cavity method.

Now, having introduced the cavity method in classical statistical mechanics we go on to generalize it to quantum mechanics. 


\section{QUANTUM CAVITY METHOD}

\section{A. Exact Framework}

We consider the modification of the Hamiltonian (1) due to the introduction of a transverse magnetic field

$$
H=-\sum_{(i j)} J_{i j} \sigma_{i}^{z} \sigma_{j}^{z}-B_{t} \sum_{i} \sigma_{i}^{x} .
$$

This is called the transverse field Ising spin glass in the literature. The Ising variables of the previous section have been replaced by Pauli matrices $\sigma^{z}$ and the magnetic field couples to the matrices $\sigma^{x}$. The fact that $\sigma^{z}$ and $\sigma^{x}$ do not commute gives rise to a host of interesting new features due to the interplay of quantum mechanics and disorder 24 .

The usual Suzuki-Trotter decomposition allows us to rewrite the problem in terms of $N_{t}$ Ising spins per quantum spin, where the number $N_{t}$ needs to be sent to infinity eventually. The additional dimension which is introduced in this way is the usual imaginary time. The $\sigma_{i}^{z} \sigma_{j}^{z}$ interactions are time-translation invariant (the disorder is correlated in the time direction) while the $\sigma^{x}$ terms give a ferromagnetic nearest-neighbor interaction in the time direction. Before writing the Hamiltonian let us introduce some notation.

For any finite $N_{t}$ we will refer to the Ising spin configuration at a given site $i$ as a "rod" of spins. The rod at site $i$ is described by $N_{t}$ spins $\sigma_{i}(t)$ where $t$ takes values from 0 to $\beta$ in steps of $\Delta t=\beta / N_{t}$, with periodic boundary conditions $\sigma(0)=\sigma(\beta)$. This notation is convenient if the limit $N_{t} \rightarrow \infty$ is eventually performed, since the rod is represented by a function $\sigma(t):[0, \beta] \rightarrow\{-1,1\}$ with $\sigma_{i}(0)=\sigma_{i}(\beta)$. The rod statistics are described by a

probability distribution $\psi[\sigma(t)]$, a functional of $\sigma(t)$, that gives a positive real number for every configuration $\sigma(t)$. The normalization condition reads $\sum_{\{\sigma(t)\}} \psi[\sigma(t)]=1$.

The partition function is written as

$$
Z=\sum_{\left\{\sigma_{i}(t)\right\}} e^{-\beta H[\sigma]}
$$

where the Hamiltonian is:

$$
\beta H=-\sum_{t} \sum_{(i j)} \Delta t J_{i j} \sigma_{i}(t) \sigma_{j}(t)-\Gamma \sum_{t} \sum_{i} \sigma_{i}(t) \sigma_{i}(t+\Delta t) .
$$

We can also write this as a sum over links of the energy of a link:

$$
\beta H_{i j}=-\sum_{t} \Delta t J_{i j} \sigma_{i}(t) \sigma_{j}(t)-\frac{1}{q} \Gamma \sum_{t} \sigma_{i}(t) \sigma_{i}(t+\Delta t)
$$


where a fraction $1 / q$ of the imaginary-time interaction is associated to each link (there are $q$ links per spin). Here $\Gamma=\frac{1}{2} \ln \operatorname{coth}\left(\beta B_{t} / N_{t}\right)$.

Notice that $\Gamma>0$ so the system is ferromagnetic, and moreover when $\beta B_{t} / N_{t} \ll 1$ then $\Gamma \gg 1$ and the coupling along the time direction is strongly ferromagnetic. In particular, for $B_{t}=0$ the spins in any given rod are locked together as $N_{t}$ useless copies of a single Ising spin. Thus, the results reduce to the classical case smoothly.

The spatial tree-like structure of the original problem is reflected in the tree-like structure of the interaction between rods. We can therefore imagine an iteration process with rods replacing the spins, in which we have $q-1$ cavity rods $\psi_{i}\left[\sigma_{i}(t)\right]$ which are merged and determine the state of the rod $\psi_{0}\left[\sigma_{0}(t)\right]$ (see Fig. 2). This corresponds to a recursion relation for the calculation of the partition function of the branches, analogous to the classical equation (6):

$$
\psi_{0}\left[\sigma_{0}(t)\right]=\frac{1}{Z} \sum_{\left\{\sigma_{i \geq 1}(t)\right\}} e^{-\sum_{i=1}^{q-1} \beta H_{0 i}} \prod_{i=1}^{q-1} \psi_{i}\left[\sigma_{i}(t)\right] .
$$

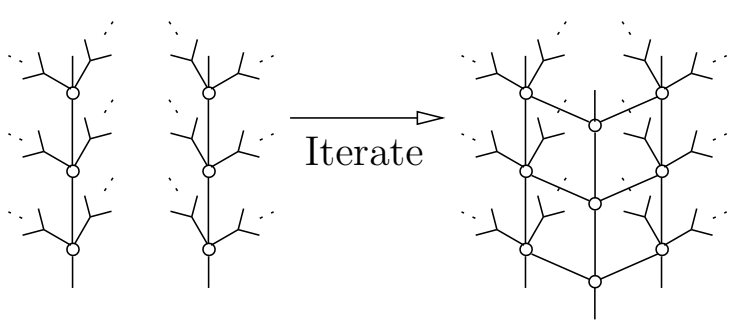

FIG. 2: Iteration of cavity rods. There are periodic boundary conditions in the imaginary time (vertical) direction. Other cavity operations are analogous.

Just as in the classical case, this iteration equation is already enough to solve an interesting ferromagnetic problem. Consider the case $q=2$ and $J_{i j}=J$. For $q=2$ our Bethe lattice is a simple chain of spins, moreover all $J$ 's being equal we can take $J>0$ without loss of generality. We then recover the well known ferromagnetic Ising chain with a transverse field, an exactly solvable system. One way to solve it is to use Onsager's transfer matrix method. In fact, the iteration equation (21) can be rewritten as just such a transfer matrix equation where $\psi$ is the $2^{N}$ dimensional vector and $e^{-\beta H_{01}}$ the transfer matrix $T$ :

$$
Z \psi_{0}=T \cdot \psi_{1} .
$$


The fixed point of this iteration gives the eigenvector $\psi$ corresponding to the largest eigenvalue $Z$. In the limit $N_{t} \rightarrow \infty$, this is an exact solution which contains all the information about classical and quantum phase transitions.

Returning to the case of the spin glass, we can write down the quantum cavity fixed point equation analogous to the classical equation (11) immediately:

$$
\begin{aligned}
P_{F P}[\psi[\sigma(t)]] & =\left\langle\delta\left[\psi[\sigma(t)]-\psi_{0}\left[\sigma(t) ;\left\{J_{0 i}, \psi_{i}\right\}_{i=1}^{q-1}\right]\right]\right\rangle_{J_{0 i}, \psi_{i}} \\
& =\int\left(\prod_{i=1}^{q-1} D \psi_{i} P_{F P}\left[\psi_{i}\right] d J_{0 i} P\left(J_{0 i}\right)\right) \delta\left[\psi[\sigma(t)]-\psi_{0}\left[\sigma(t) ;\left\{J_{0 i}, \psi_{i}\right\}_{i=1}^{q-1}\right]\right]
\end{aligned}
$$

where the iterated rod action $\psi_{0}\left[\sigma(t) ;\left\{J_{0 i}, \psi_{i}\right\}_{i=1}^{q-1}\right]$ is given by Eq. (21) and $P\left(J_{0 i}\right)$ is the fixed prior distribution for couplings Eq. (10). This is a functional equation for $P_{F P}[\psi[\sigma(t)]]$, the fixed point probability distribution of the effective distribution describing iterated cavity rods. In the limit $N_{t} \rightarrow \infty$, it is exact but difficult to solve in closed form. It is certainly possible that analytic progress can be made, but we have not succeeded thus far. However, it is amenable to numerical study at finite $N_{t}$ under certain approximations and also perhaps by continuous time Monte Carlo for $N_{t} \rightarrow \infty$. In the remainder of this section we will explore the finite $N_{t}$ approach.

We must first parameterize our generic vector $\psi$ in the $2^{N_{t}}$-dimensional space of the configurations of the rods. In principle it is described by $2^{N_{t}}-1$ real numbers, which can be reduced by a factor $O\left(N_{t}\right)$ by exploiting time-translation symmetry and the periodic boundary conditions. A natural way to parameterize it is in term of the effective action of the rod

$$
\psi[\sigma(t)]=e^{-S[\sigma]}
$$

where we expand $S$ in a series of increasing clusters of interacting spins.

$$
\begin{aligned}
S[\sigma]= & -\log Z-h \Delta t \sum_{t} \sigma(t)-\sum_{t, t^{\prime}} \Delta t^{2} C^{(2)}\left(t^{\prime}-t\right) \sigma(t) \sigma\left(t^{\prime}\right)- \\
& -\sum_{t, t^{\prime}, t^{\prime \prime}} \Delta t^{3} C^{(3)}\left(t^{\prime}-t, t^{\prime \prime}-t^{\prime}\right) \sigma(t) \sigma\left(t^{\prime}\right) \sigma\left(t^{\prime \prime}\right)+\ldots
\end{aligned}
$$

In principle, the sum includes up to $N_{t}$-spin interaction terms (the normalization factor has been included as a spin-independent term in the effective action). In practice, we truncate the action expansion at second order to keep the numerical requirements manageable. We comment below on the limits in which this truncation is exact. Notice that $C^{(2)}(t)$ is the 
kernel for 2-point in time interactions, not the dynamical two-point correlation function, often denoted $c^{(2)}(t)=\langle\sigma(t) \sigma(0)\rangle$.

The functions $h, C^{(i)}$ are random quantities characterized by the Markov process defined by the iteration procedure. By writing the representations of the vectors $\psi$ in terms of the effective action (25) we can rewrite the iteration equation as

$$
e^{-S\left[\sigma,\left\{h_{0}, C_{0}\right\}\right]}=\sum_{\left\{\sigma_{1}(t)\right\}, \ldots,\left\{\sigma_{q-1}(t)\right\}} e^{-\sum_{i=1}^{q-1} \beta H_{0 i}} \prod_{j=1}^{q-1} e^{-S\left[\sigma,\left\{h_{j}, C_{j}\right\}\right]} .
$$

This gives an implicit update map from the 'old' $q-1$ parameters $h_{j}, C_{j}^{(2)}, C_{j}^{(3)}, \ldots$ and the couplings $J_{0 j}, B_{t}$ to the 'new' parameters $h_{0}, C_{0}^{(2)}, C_{0}^{(3)}, \ldots$ The statistics generated by this Markov process and in particular its fixed point distribution

$$
P\left(h, C^{(2)}, C^{(3)}, \ldots\right)
$$

are the solution of the problem.

\section{B. Approximations}

\section{Action Representation}

By parameterizing the quantum dynamics through the action expansion (25) we do not make any a priori assumptions about the nature of the spin-spin correlations in the time direction - that is, if we could keep all of the terms in the expansion, it would be an exact treatment. We avoid in this way the spherical approximation which has been used in Ref. 20, since the results thus obtained do not reduce to the well-known classical results for $B_{t}=0$.

In practice, however, we truncate the cavity actions to second order. Most usefully, this corresponds to the leading order term in a large connectivity expansion of the effective action. Indeed, in a large $q$ treatment, in which the couplings $J_{i j}$ must be scaled as $1 / \sqrt{q}$ for the disordered model, one finds that the one- and two-body terms in the effective action for the rod at the root of a tree are $\mathcal{O}\left(1 / q^{0}\right)$, the three- and four-body terms are $\mathcal{O}\left(1 / q^{1}\right)$ and so on 22 . The truncation to second order is thus both exact and necessary in the $q \rightarrow \infty$ limit. We note that the oft-used static approximation ${ }^{26}$ is thus incorrect even at infinite connectivity for disordered models. The truncation to second order is also exact at high temperature, regardless of the value of $q$. 
Numerical investigations of small systems suggest that the higher order interactions are quantitatively small more generally, even at $q=3$. This is especially true at small $B_{t}$, where the strong ferromagnetism in the time-like nearest neighbor bonds dominates. We note that there is also some error in the numerical fitting of an iterated cavity distribution to a truncated action. This problem is reminiscent of a maximum entropy model for the statistics of signals and the inverse Ising problem of computer science 27 . However, even in the disordered system, the interaction of the spins along imaginary time is always ferromagnetic and the problem does not present the difficulties that usually accompany the inverse problems in general statistical mechanics. In other words, although the original problem is

fundamentally frustrated, none of this frustration appears in the single spin dynamics. The frustration is taken care of in the treatment of the relevant parameters $h, C^{(i)}$ as random numbers.

For comparison, we note that our approach is closely related to self-consistent dynamical mean field theory (DMFT) methods, which also truncate to non-local two point interactions in the single-spin effective action (these are the so-called Weiss functions or bare Green's functions of DMFT treatments). However, DMFT techniques cannot be used straightforwardly for disordered systems. One cannot assume that the imaginary-time propagator (or action) for a spin is equal to that of the spins which surround it but rather that they come from the same probability distribution. If this observation is taken into account then we expect to recover functional equations for the distribution of single site Green's functions analogous to those we have proposed for the single site action.

\section{Finite Discretization Error}

Finally, we comment on where in the $\left(\tau, B_{t}\right)$ phase diagram the fixed $N_{t}$ approach will give reliable results. As mentioned above, on the classical line $B_{t}=0$, all spins in a rod are locked together by divergent nearest neighbor interactions and the Trotter decomposed system (for any $N_{t}$ ) reduces to the classical system exactly. In the opposite limit of large $B_{t}$ at fixed $N_{t}$, the planes of the Trotter system decouple as $\Gamma \rightarrow 0$. Each of these planes is an exact copy of the original $B_{t}=0$ spin glass model except with couplings $J_{i j} / N_{t}$. These will therefore undergo independent thermal phase transitions at $\tau=\tau_{c} / N_{t}$ and no $\tau=0$ critical field will be detected. This explains the phenomenon of "asymptotic critical lines" that we 
note in our finite $N_{t}$ phase diagrams.

More generally, for the finite $N_{t}$ approximation to be valid, $N_{t}>k_{\text {typ }}$ where $k_{\text {typ }}$ is the typical number of kinks in a rod. For a single spin in a transverse field, a straightforward calculation shows $\langle k\rangle=2 \beta B_{t} \tanh \beta B_{t}$, which for large $\beta B_{t}$ reduces to $\langle k\rangle \approx 2 \beta B_{t}$. Within the paramagnetic phase this calculation remains nearly exact, although in the spin glass phase $\langle k\rangle$ will be suppressed by the presence of longitudinal cavity fields. However, this inequality $N_{t}>2 \beta B_{t}$ remains a good indicator of the quality of the approximation and agrees with the regions where it clearly breaks down.

Thus, this expansion is particularly useful close to line $B_{t}=0$, and we will see that it gives reasonable results and insights into the structure of the problem also deep in the quantum spin glass phase. On the other hand, the other region of interest $T=0, B_{t} \simeq B_{t}^{\text {crit }}$ should not be addressed with this expansion, unless the description of the spins in terms of continuous time functions $\sigma(t)$ turns out to be treatable in the future. In this paper, we will not be able to make definitive statements about the nature of the quantum phase transition which occurs at this point but we are definitely able to make statements about the nature of the spin glass phase when quantum effects are not negligible.

In the next section we show how a simple minded Trotter discretization with $N_{t}$ relatively small ( $N_{t}=6$ to 11$)$ delivers a great deal of information about the quantum spin glass phase.

\section{NUMERICAL RESULTS}

We solve the fixed point equation (23) numerically using a population dynamics algorithm analogous to that of Mezard and Parisi $\frac{15}{}$. We represent $P[\psi]$ by a finite population of $N_{\text {rods }}$ $\operatorname{rod}$ actions $\left\{\psi_{i}[\sigma(t)]=e^{-S_{i}[\sigma(t)]}\right\}$, where the expansion (25) of $S_{i}$ is truncated to second order. Each rod action is therefore specified by $1+\left\lfloor\frac{N_{t}}{2}\right\rfloor$ distinct numbers $\left(h, C^{(2)}(t)\right)$ after exploiting the periodicity of imaginary time.

This population is initialized from an appropriate uniform distribution and then iterated as follows:

1. Select $q-1$ rods $\psi_{i}$ randomly from the population and $q-1$ random $J_{0 i}$.

2. Use (21) to calculate the effective action on an iterated cavity spin $\psi_{0}$ from these rods. In principle, higher order interactions may be generated but we truncate them 
by finding the second order action that exactly reproduces the free energies of a series of domain wall configurations of varying width.

3. Randomly replace one element of the population with $\psi_{0}$.

4. Repeat until convergence in some measure of the population, for example the order parameter $q_{E A}$.

In practice, this procedure converges quickly deep in either the glassy or paramagnetic phase but slows near the phase transition as the flow of $P[\psi]$ under the iteration equation near the paramagnetic fixed point becomes marginal.

Given $P_{F P}[\psi]$, we can calculate the sample averaged free energy density and local observables such as the link energy, site magnetization, Edwards-Anderson order parameter

$q_{E A}=\left\langle\left\langle\sigma_{i}\right\rangle_{T}^{2}\right\rangle_{i}$ and reduced von Neumann entropy $S_{\text {von } N}=-\operatorname{tr} \rho_{0} \log _{2}\left(\rho_{0}\right)$ by standard Monte Carlo sampling of these quantities. The free energy density is given by equation (16). The reduced entropy and transverse magnetization may be derived in the usual way from the reduced density matrix $\rho_{0}$ for a spin. We calculate this by performing a merge operation onto a "broken rod" (see Fig. [3), in which periodic boundary conditions are not enforced. The various elements of the reduced density matrix correspond to imposing different values on the top and bottom spin of the broken rod and summing out all other spins in the partition function. Finally, calculating the average internal energy can be done by averaging the Hamiltonian (17).

\section{A. Numerical Results}

\section{Phase Diagram}

We present numerical results for an investigation of the $q=3$ connectivity model using a naive (exact) approach to the exponential summation involved in the cavity iteration and merging operations $\frac{36}{6}$ Fig. $4\left(\right.$ a) shows the phase diagram calculated at $N_{t}=10, N_{\text {rods }}=$ $2500, N_{\text {iter }} \sim 1000 N_{\text {rods }}$ and suggestively fit to $N_{t} \rightarrow \infty$ using asymptotic expansions in $1 / N_{t}^{2}$. Qualitatively, all is as might be expected:

- At any $N_{t}$, the phase transition curve predicts a $B_{t}=0$ critical temperature in agreement with the analytic prediction of $\tau_{c}=1 / \tanh ^{-1}(1 / \sqrt{q-1}) \approx 1.13$. 


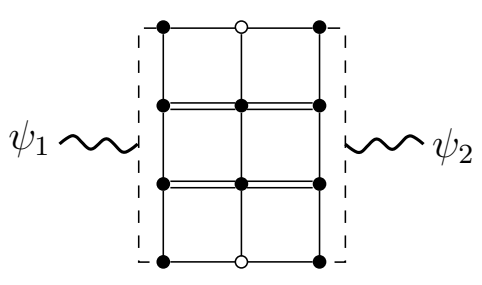

FIG. 3: Merging of two cavity rods onto a "broken" central rod. Here $N_{t}=3$ but instead of imposing periodic boundary conditions everywhere we depict $\sigma(0)$ and $\sigma\left(N_{t}\right)$ as independent spins. Each vertical link corresponds to a $\Gamma$ coupling while each horizontal link corresponds to a $J_{i j} / 2 N_{t}$ coupling. The dashed lines connecting the cavity rods indicate identification of the top and bottom spins for each rod and the wiggly line indicates an effective action. By imposing different values on the top and bottom spin of the broken rod and summing out the rest, the elements of the reduced density matrix $\rho_{0}$ may be determined.

- The upturn in the $N_{t}=10$ phase boundary at low temperature is due to the finite discretization of time, which leads to an asymptotic phase transition line at $\tau=\frac{\tau_{c}}{N_{t}} \approx$ 0.113 .

- While the fits to $N_{t} \rightarrow \infty$ are certainly approximate, we believe that the true $\tau=0$ critical field lies between 1.5 and 2. We believe continuous time techniques will allow dramatic refinement of this estimate and investigation of the quantum critical region.

Our phase diagram clearly disagrees with that of Kopec and Usadel ${ }^{20}$, who treat the identical model using a soft spherical approximation and find that both the critical temperature and critical transverse field are depressed relative to our values. Presumably this suppression of ordering arises due to the stronger effect of fluctuations in the softened model.

Figure 4(b) shows the instance averaged single site von Neumann entropy $S_{v o n N}$ which has a remarkably clear maximum near the phase transition curve above the classical line. This reflects the strength of quantum correlations even at the finite temperature phase transition. See Ref. 28 for discussion of local measures of entanglement at finite temperature.

Zooming in on the horizontal stripe at $B_{t}=1$ indicated on the phase diagram, we find that $q_{E A}$ vanishes linearly at the critical temperature (Fig. 5(a)). This reflects the underlying broadening transition in $P[\psi]$, which can be seen sharply in the variances of each of the effective action coefficients (Figs. [5(b,c)). We use this behaviour to estimate 
sharp transition points despite softening due to critical slowing in the convergence of our procedure.

Finally, we note that much of the phase diagram is surprisingly stable to variation in $N_{t}$. We have explored various regions of the phase space at $N_{t}=6,7,8,9,10,11$. The classical line $\left(B_{t}=0\right)$ at all temperatures is completely stable down to $N_{t}=1$ as expected. Perhaps more surprisingly, moving between $N_{t}=8$ and $N_{t}=10, q_{E A}$ is essentially stable below $B_{t}=1$ down to temperatures $\tau \sim 0.15$. Of course, the high field, low temperature part of the phase transition curve moves downward as the finite discretization asymptote goes towards the $\tau=0$ axis. See Fig. 6 for the low temperature critical curves estimated using vertical stripes run at five different temperatures (corresponding to $\beta=3.5,4,4.5,5,5.5$ ) at various $N_{t}$.
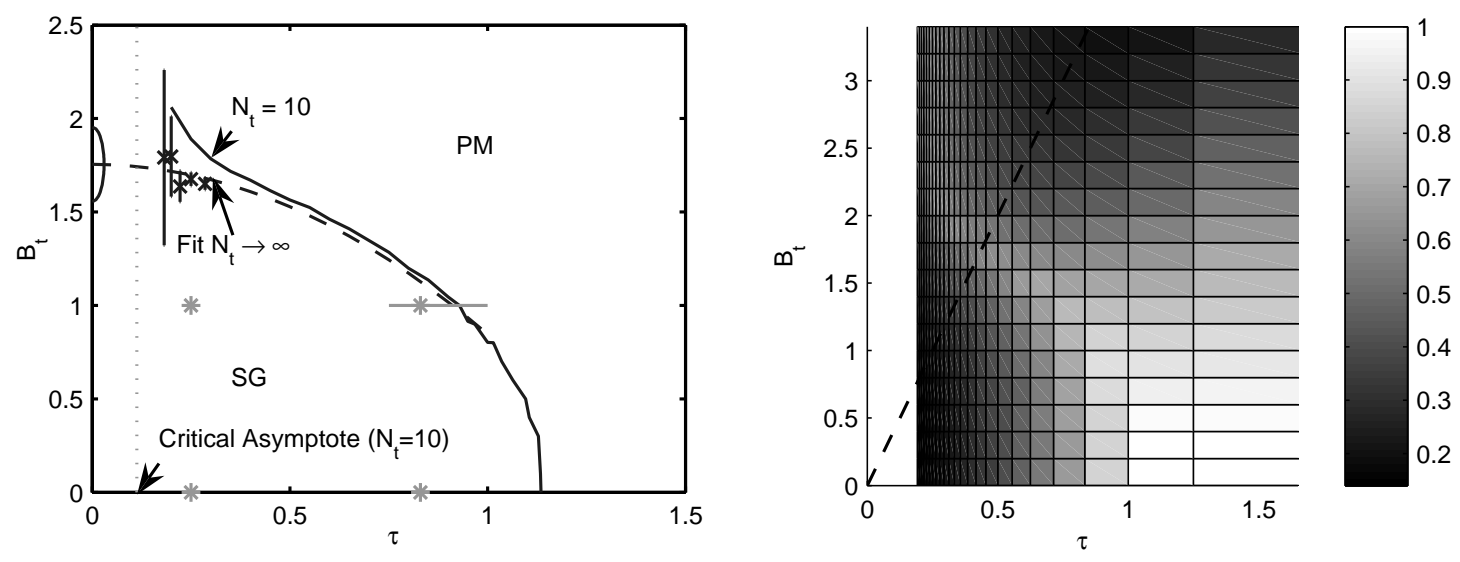

FIG. 4: (a) Phase diagram at $q=3$. The solid phase transition curve has been calculated at $N_{t}=10, N_{\text {rods }}=2500, N_{\text {iter }}=1000 N_{\text {rods }}$ on a fine mesh in the $\left(\tau, B_{t}\right)$ plane. The vertical dotted line is the asymptotic critical line for large $B_{t}$ at $N_{t}=10\left(\right.$ ie $\left.\tau=\tau_{c} / N_{t}\right)$. The points marked $\mathrm{x}$ with error bars indicate $N_{t} \rightarrow \infty$ fits based on Fig. 6. The dashed transition curve is a weighted quadratic fit through the estimated low temperature points and the $N_{t}=10$ points in the range $0.5<\tau<1$. This leads to an estimated $B_{t}^{c}=1.775 \pm 0.03$. As this fit is clearly heuristic, we have suggested a much larger range for our estimate of $B_{t}^{c}$ in the Figure. The stars and stripes indicate points in the phase space which we have investigated in more detail below. (b) The average von Neumann entropy $S_{v o n N}$ (in bits) of a central spin as a function of $\left(\tau, B_{t}\right)$ at $N_{t}=8$. The dashed line indicates the estimated region of validity of the discretization approximation $\left(B_{t} \leq N_{t} \tau / 2\right)$. 

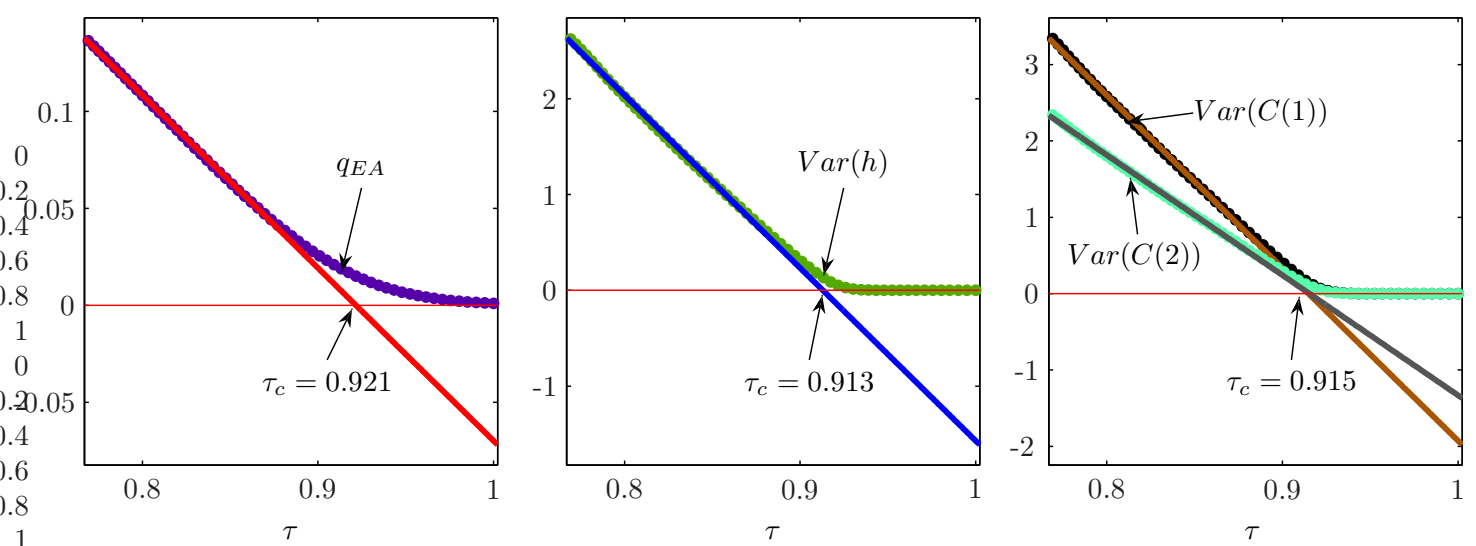

FIG. 5: These graphs all correspond to the horizontal slice at $B_{t}=1$ shown in Fig. 4. The order parameter $q_{E A}$ and all effective action variances undergo mean field like transitions at the critical temperature (eg. $\left.q_{E A} \sim\left|\tau-\tau_{c}\right|^{1}\right)$. This allows us to estimate the critical temperature precisely despite softening due to critical slowing down near the phase transition. These curves were calculated at $N_{t}=8$. However, the results are stable to increasing $N_{t}$ to 10 to within an error of \pm 0.005 in $\tau_{c}$.

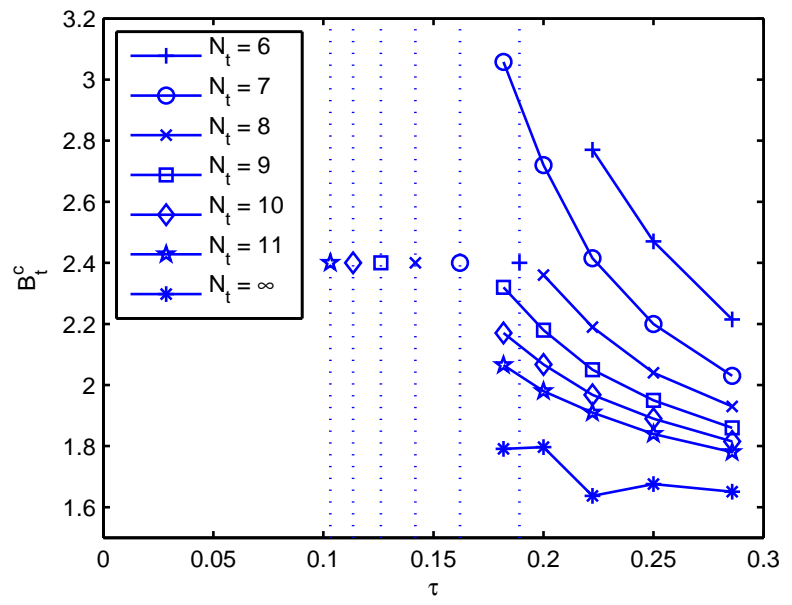

FIG. 6: The phase transition curve in the high field, low temperature regime at various $N_{t}$. The vertical dashed lines indicate the finite discretization critical asymptotes. The estimated curve for $N_{t} \rightarrow \infty$ in Fig. 4a is given by fitting $B_{t}\left(N_{t}\right)=a / N_{t}^{2}+b / N_{t}^{4}+c$ to the points calculated at each temperature and then sending $N_{t}$ to infinity in the result. These fits suffer from a paucity of data, but are suggestive nonetheless. 


\section{Structure of the Glassy Phase}

Since the order parameter is a probability distribution of an action, there is a rich structure to be investigated at even a single point $\left(\tau, B_{t}\right)$ within the glassy phase. In Fig. 7 the marginal probability distribution of the field term $h$ in the cavity action is shown at the four points indicated on Fig. 4(a). The two lower distributions lie on the classical line $\left(B_{t}=0\right)$, one deep within the glassy phase and one near the transition. It is clear that the distinctive features of the classical solution are reproduced here: a Gaussian-like structure around $h=0$ near the phase transition with the appearance of delta function spikes on the integer fields deep within the phase. At $B_{t}=1$, the qualitative picture of spread from narrow Gaussian near the phase transition to broader, bumpier distribution remains. It is less clear whether the sharply defined spikes on integer fields would remain at $\tau=0$ with large $B_{t}$.

Further structure can be found in the nontrivial probability distribution for the interaction terms that develop in the spin glass phase. Figure 8 shows the histogram for various marginal and $h$-conditioned distributions of the nearest neighbor and next nearest neighbor interactions terms at $\left(\tau=0.25, B_{t}=1\right)$ (cf. Fig. 7 (top left)). We can qualitatively understand many features of these distributions:

- The two-spin interactions are ferromagnetic and the effect of coupling to neighboring rods is only to enhance the ferromagnetic interaction from the bare nearest neighbor interaction on a single rod $(\Gamma)$. Indeed, this $\Gamma$ sets the minimum strength of $C(\delta T)$, as can be seen in the top row.

- The strength of two-spin interactions are strongly anticorrelated with the strength of the cavity field $h$ as can be seen from the decomposition of the full marginal distributions of $C(\delta t)$ and $C(2 \delta t)$ into the small (middle column) and large (right column) cavity field conditioned distributions. Large cavity fields on a central spin come from large fields biasing neighboring rods. These fields pin the neighboring spins more strongly and reduce the ability of those spins to mediate interactions in time between the central rod spins, reducing the effective two-spin interaction.

- The multimodal spikiness in these distributions reflect the spikiness in the low temperature cavity field distributions through the field-interaction correlation. 
Unfortunately, we have yet to develop a more significant analytic understanding of these correlations nor a means to extrapolate them to zero temperature in the presence of the transverse field.

Finally, we emphasize that the phase transition is signaled by a singular broadening of $P$ rather than any singularity in its first moments or in the structure of the typical imaginary time action. This can be seen in the smooth evolution of $\langle C(2 \Delta t)\rangle_{J}$ through the phase transition in Fig. 9a and the similarly smooth evolution of the average two point correlation function $\langle\sigma(0) \sigma(t)\rangle_{J}$. This is in contrast to the ferromagnetic case in which the distribution $P(\psi)$ would exhibit spontaneous symmetry breaking but remain deterministic.
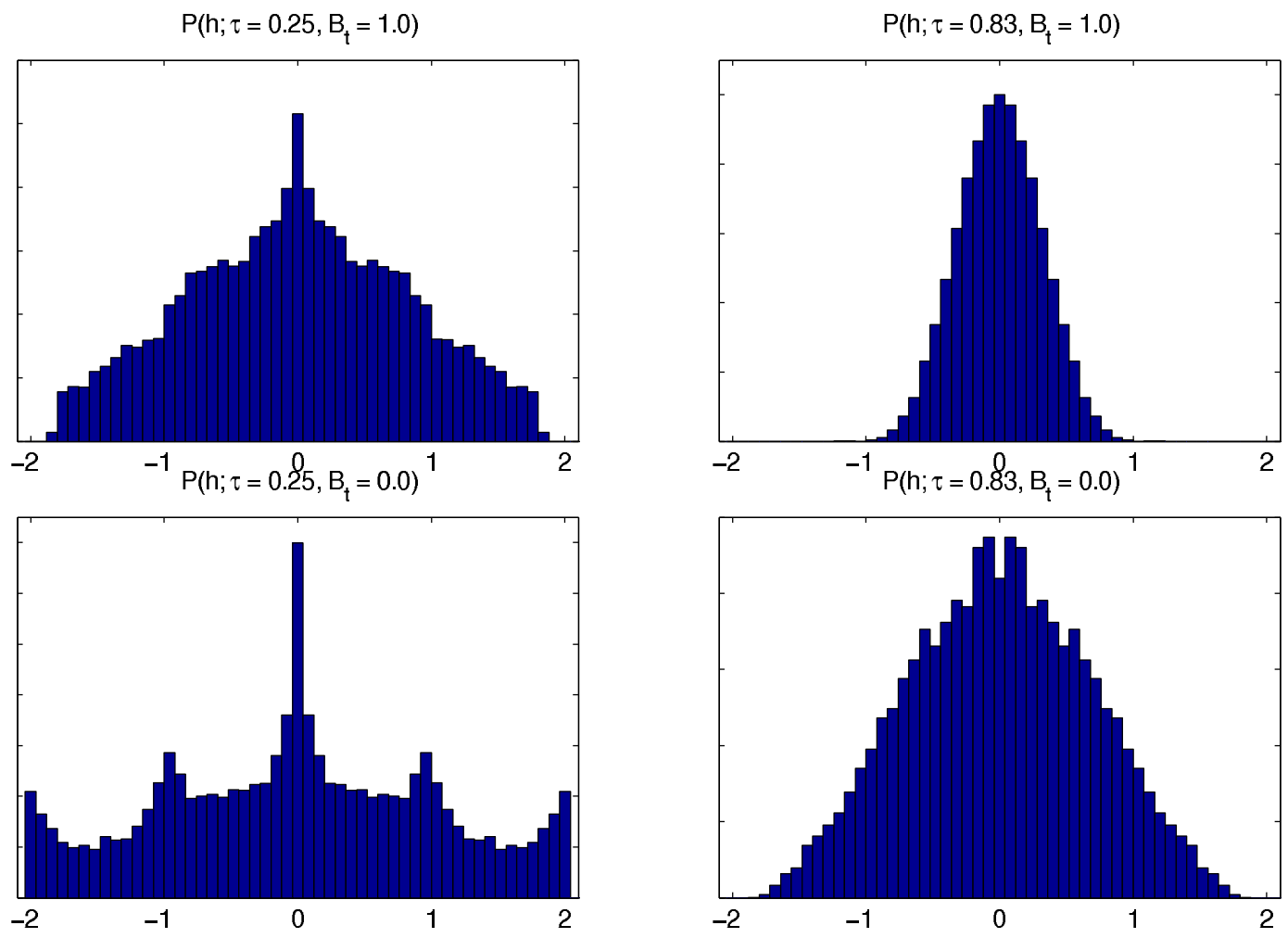

FIG. 7: The distribution of the field term of the cavity rod action at the four different points in phase space labeled by the stars on Fig. 4, The distinctive distribution of the low temperature replica symmetric classical spin glass is reproduced in the bottom left corner while the three other points all lie closer to the phase boundary in the $\tau$ or $B_{t}$ directions. 

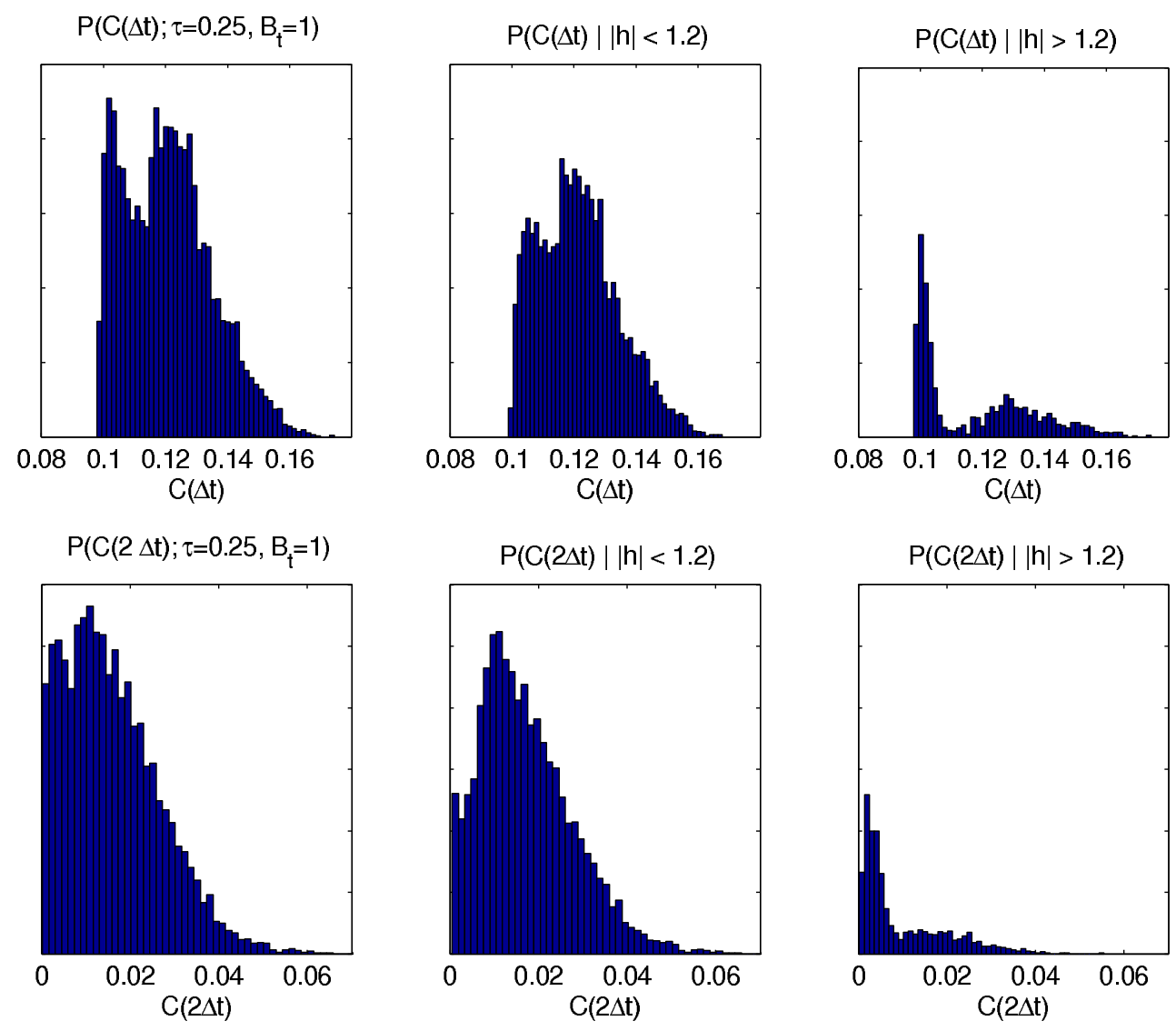

FIG. 8: Histograms of nearest neighbor (top row) and next nearest neighbor (bottom row) interactions in imaginary time at $\tau=0.25, B_{t}=1$ (in the SG phase). The second and third column provide the conditional distribution of the interactions given $|h|$ is small or large.

\section{DISCUSSION AND FURTHER WORK}

The cavity method has a long and illustrious history in the study of statistical systems from Bethe's early work on the Ising ferromagnet to modern studies of random constraint satisfaction problems in computer science. In this paper, we have introduced a new variant for studying disordered quantum systems within an imaginary time formalism. This represents an intuitively appealing, natural synthesis of the classical disordered model techniques with the quantum homogeneous models studied in DMFT. We note that our framework can be simply adapted to study many other transverse field Ising systems on trees with fixed or fluctuating connectivity - such as the ferromagnet, diluted ferromagnet or biased glass.

We have shown that the transverse field Ising glass on a Bethe lattice of connectivity 

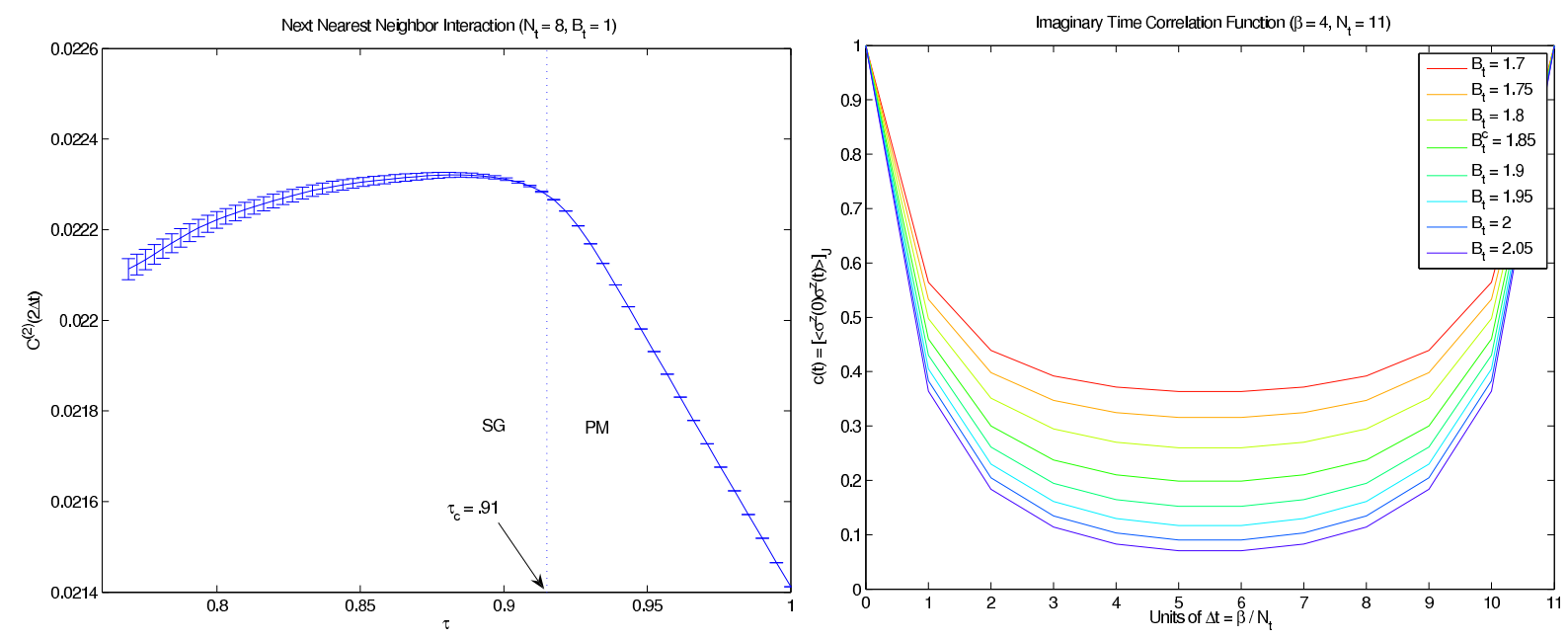

FIG. 9: (a) The average next to nearest neighbor interaction in time $C^{(2)}(2 \Delta t)$ of a cavity rod action at $B_{t}=1$, varying the temperature. The bars indicate the variance of $C^{(2)}(2 \Delta t)$. Notice the zero variance above the critical temperature. (b) Single site imaginary time correlation function $\langle\sigma(0) \sigma(t)\rangle_{J}$ at $\tau=0.25$ for various $B_{t}$ passing through the transition at $B_{t}^{c} \sim 1.85$.

three has a phase transition line all the way from the classical $B_{t}=0, T=1.13$ to the quantum $B_{t} \approx 1.75, T=0$. At finite temperature, the transition is classical and mean field like. Inside the frozen phase the picture is similar to the classical case: when a randomly chosen spin is extracted from the graph, its effective action (analogous to the cavity field) is well-defined in the paramagnetic region but is a random functional in the spin-glass phase. In this phase all of the local observables, classical and quantum, are therefore also random variables. In principle one could also study the properties of the entanglement of distant spins, which cannot be done in the better known but fully connected SK model.

The reader familiar with classical spin glass theory will have noticed our avoidance of the important issue of replica symmetry breaking (RSB), which is widely believed to be a feature of a correct treatment of the random graph Bethe lattice. In this connection, we note that our simpler treatment is indeed correct for models on Cayley trees with fixed boundary conditions. Quantum RSB phenomena certainly deserve further study: the conceptual difficulties of the RSB ansatz become even thornier in the presence of quantum tunneling. We note that formally breaking replica symmetry at the one step level (1RSB) should be straightforward in our framework. In analogy with Ref. 15, one should introduce populations of populations of effective actions and weigh them according to their free energy (as 
one does for different solutions of the TAP equations). This straightforward modification of the algorithm makes it computationally considerably more time consuming. For this first pass, we decided not to embark on such a project.

Unfortunately, we are not aware of Quantum Monte Carlo or other numerical studies on the transverse Ising model on the Bethe lattice with which to compare our results. The primary difficulty that has prevented the direct simulation of this system is that it requires a very large number of spins to approximate the infinite system effectively. Since a random graph with fixed connectivity has an extensive number of loops of lengths $\ell \sim$ $\mathcal{O}(\ln N / \ln (q-1)), N$ needs to be exponentially larger than any statistically relevant length scale (e.g. the coherence length).

Another direction for further development would be to find a spin glass (or otherwise) model amenable to analytic treatment within the quantum cavity method. A "soft spin" Gaussian model would do, since the path integrals to be performed in an iteration could be computed exactly. However, we do not believe this model has a spin glass phase in the absence of higher order couplings. Whether one could treat such a coupling perturbatively is a question worthy of further exploration.

\section{ACKNOWLEDGMENTS}

We would like to thank M. Aizenmann, R. Bhatt, D. Huse for discussions. C.L. and A.S. would like to thank G. Santoro, R. Fazio, E. Tosatti and R. Zecchina for their kind hospitality at ICTP and SISSA in Trieste, where part of this work was completed, and for discussions. A.S. would like to thank E. Farhi and J. Goldstone for discussions during the early stages of this project.

Note added: During the production of this manuscript, several other groups 29,30 have independently developed quantum cavity techniques inspired by somewhat different approaches to the quantum problem. Ref. 31 provides a quantitative comparison of the error in several of these related techniques. In an important development, Ref. 32 describes a continuous time version of the quantum cavity method described here which they apply to 
a ferromagnetic model.

1 D. Sherrington and S. Kirkpatrick, Phys. Rev. Lett. 35, 1792 (1975).

2 D.J. Thouless, P.W. Anderson and R.G. Palmer, Philos. Mag. 35, 593 (1977).

3 A.J. Bray and M.A. Moore, J. Phys. C 13, L469 (1980).

4 J.R.L. de Almeida and D.J. Thouless, J. Phys. A 11, 983 (1978).

5 G. Parisi, J. Phys. A 13, L115 (1980); 13, 1101 (1980); 131887 (1980); Phys. Rev. Lett. 50, 1946 (1983).

6 P.W. Anderson, Lectures on Amorphous Systems, 31st Session of the Les Houches Summer School, Les Houches, France, July-Aug. 1978. pp. 159-261.

7 D. R. Bowman and K. Levin, Phys. Rev. B 25, 3438 (1982).

8 L. Viana and A.J. Bray, J. Phys. C 18, 3037 (1985).

9 J.T. Chayes, L. Chayes, J.P. Sethna and D.J. Thouless, Commun. Math. Phys. 106, 41 (1986); J.M. Carlson, J.T. Chayes, L. Chayes, J.P. Sethna and D.J. Thouless, Europhys. Lett. 5, 355 (1988).

10 C. Kwon and D.J. Thouless, Phys. Rev. B 37, 7649 (1988).

11 P.Y. Lai and Y.Y. Goldschmidt, J. Phys. A 22, 399 (1989); Y.Y. Goldschmidt and P.Y. Lai, J. Phys. A 23, L775 (1990).

12 D.S. Fisher and D.A. Huse, Phys. Rev. Lett. 56, 1601 (1986); Phys. Rev. B 38, 386 (1988).

13 R. Monasson, R. Zecchina, S. Kirkpatrick, B. Selman and L. Troyansky, Nature 400, 133 (1999); M. Mezard, G. Parisi and R. Zecchina, Science 297, 512 (2002); M. Mézard and R. Zecchina, Phys. Rev. E 66, 56126 (2002).

14 B. Bollobas, Random Graphs, (Cambridge Univ. Press, Cambridge 2001).

15 M. Mezard and G. Parisi, J. Stat. Phys. 111, 1 (2003); Eur. Phys. J. B 20, 217 (2000).

16 S. Kirkpatrick, C.D. Gelatt Jr and M.P. Vecchi, Science 220, 671 (1983).

17 Y. Fu and P. Anderson, J. Phys. A 191605 (1986).

18 A. Montanari, F. Ricci-Tersenghi and G. Semerjian, J. Stat. Mech. 2008, 04004 (2008).

19 E. Farhi, J. Goldstone, S. Gutmann, J. Lapan, A. Lundgren and D. Preda, Science 292, 472 (2001).

20 T.K. Kopec and K.D. Usadel Phys. Rev. Lett. 78, 1988 (1997); Phys. Stat. Sol. (b) 243, No. 
$2,502(2006)$.

21 S. Knysh and V.N. Smelyanskiy Arxiv preprint cond-mat/0602257; A. Boulatov and V.N. Smelyanskiy, Phys. Rev. A, 68, 62321 (2003).

22 A. Georges, G. Kotliar, W. Krauth and M.J. Rozenberg, Rev. Mod. Phys. 68, 13 (1996).

23 S.F. Edwards and P.W. Anderson, J. Phys. F. 5, 965 (1975).

24 D.S. Fisher, Phys. Rev. B 50, 3799 (1994); B.M. McCoy and T.T. Wu, Phys. Rev. 176, 631 (1968); O. Motrunich, S-C. Mau, D. Huse and D.S. Fisher, Phys. Rev. B 61, 1160 (2000).

25 C. De Dominicis and P. Mottishaw, Journ. Phys. A 20, L1267 (1987).

26 K.D. Usadel, Solid State Commun. 58, 629 (1986); H.-J. Sommers and K.D. Usadel, Z. Phys. B 47, 63 (1982).

27 G.E. Hinton and T.J. Sejnowski in Parallel distributed processing, D.E. Rumelhart and J.L. McClelland eds., p. 282 (MIT Press, Cambridge 1986).

28 Arnesen et al. Phys. Rev. Lett. 87 (2001).

29 M.B. Hastings, Phys. Rev. B 76, 201102 (2007).

30 M. Leifer and D. Poulin, Ann. Phys. 3231899 (2008).

31 D. Poulin and E. Bilgin, Phys. Rev. A 77, 052318 (2008).

32 F. Krzakala, A. Rosso, G. Semerjian, F. Zamponi, arXiv:0807.2553 (2008).

33 It is interesting to notice that in the TAP approach 2 , the free energy was found as a consequence of the mean field equations, reversing the usual order of reasoning. This was mended by Anderson shortly thereafter ${ }^{\underline{6}}$ and in many other papers later. However this unusual, secondary role of the free energy in the problem at hand has survived until today and seems to be a peculiarity of spin glasses.

34 In particular, the cavity field distribution will depend only on the depth of a site in the Cayley tree, assuming i.i.d. boundary fields. This distribution becomes depth independent sufficiently deep in the tree.

35 Readers familiar with the literature may note that this allows one to bypass the parameterization of the order parameter on the replica indices, a particularly thankless task on the Bethe lattice since an infinite sequence of order parameters $q_{a, b}, q_{a, b, c}, q_{a, b, c, d}, \ldots$ is necessary 25 .

36 We note that a rearrangement of the sum over configurations according to the number of kinks looks like a natural perturbative expansion in $B_{t}$ for the system and naively scales polynomially $O\left(N_{t}^{k}\right)$ where $k$ is the maximum number of kinks summed. Unfortunately, little is gained from 
this rearrangement in terms of the accurately computable region of the phase diagram: the expected number of kinks for relevant rod configurations scales $\sim 2 B_{t} / \tau$ and thus the sum is again exponential in $B_{t} / \tau$. 